Scientific Research \& Studies Center-Faculty of Science- Zagarig

University - Egypt

Biochemistry Letters

Journal home page:

\title{
Pilot study for association of chemokine CCL2 rs13900 with the Susceptibility to HCV Infection
}

\author{
Youssef MM ${ }^{\text {a }}$, El-Bendary MM ${ }^{\mathrm{b}}$, Neamatallah MA ${ }^{\mathrm{c}}$, Zein MA $^{\mathrm{a}}$ \\ ${ }^{a}$ Biochemistry Department, Faculty of Science, Mansoura University, Mansoura, Egypt \\ ${ }^{\mathrm{b}}$ Tropical Medicine Department, Faculty of Medicine, Mansoura University, Mansoura, Egypt \\ ${ }^{c}$ Biochemistry Department, Faculty of Medicine, Mansoura University, Mansoura, Egypt
}

\begin{tabular}{ll}
\hline A R T I C E I N F O & A B T R A C T \\
\hline SNP, gene polymorphism, RT-PCR, & Background: In some genes, Polymorphisms may have an \\
influence on the persistence of HCV infection, HCV \\
replication, or spontaneous clearance of the virus. This study \\
was carried out to investigate the association of chemokines \\
CCL2 gene polymorphism with the susceptibility to HCV \\
infection with high-resolution techniques in Egyptian families. \\
Methods: A total of 75 Egyptian families with a total of 291 \\
subjects in this study were recruited from different Egyptian \\
populations from Dakahlia governorate and were classified into \\
2 groups: 146 infected persons with chronic hepatitis C (CHC), \\
145 subjects as a healthy control group. All subjects were \\
genotyped for rs13900 C/T SNP of CCL2 gene using allelic \\
discrimination real time PCR (RT-PCR) technique. Results: \\
The allele carriage of at least one copy of C allele of CCL2 \\
rs13900 C/T polymorphism was significantly lower in the \\
positive group when compared to that of negative group (OR $=$ \\
$0.4169,95 \%$ CI 0.1830 to $0.9496, p 0.0372)$ indicating that The \\
C allele of rs13900 C/T polymorphism is protective allele \\
against development of chronic HCV while the allele carriage \\
of homozygotes TT of CCL2 rs13900 C/T polymorphism was \\
significantly higher in the positive group when compared to \\
that of negative group (OR = 2.3986, 95\% CI 1.0530 to 5.4635, \\
p 0.0372) indicating that The C allele of rs13900 C/T \\
polymorphism is protective allele against development of \\
chronic HCV. Conclusions: HCV infection is associated with \\
rs13900 C/T SNP of CCL2 gene in the Egyptian families.
\end{tabular}

(C) 2018 Publisher All rights reserved.

Corresponding author: Youssef MM, Biochemistry Department, Faculty of Science, Mansoura University, Mansoura, Egypt 


\section{INTRODUCTION}

Hepatitis C virus (HCV) leads to acute and often chronic hepatitis ${ }^{[\underline{[l]}}$. Almost, 170 million people globally are evaluated to be infected with $\mathrm{HCV}^{[2]}$. The natural course of chronic disease is considered to have high variation and ranges from asymptomatic to mild disease to cirrhosis and hepatocellular carcinoma (HCC) and HCV-related codiseases $[\underline{3}]$.

The high prevalence of chronic $\mathrm{HCV}$ infection suggest that both the innate and the adaptive immune system is not enough to eliminate the virus for most patients ${ }^{[4]}$.

Chemokines are a huge family of tiny chemoattractant cytokines that have a crucial role in the cell trafficking regulation and in the induction of chemotaxis[5]. mRNA transcripts for CCL2 and CCR2 are significantly raised in $\mathrm{HCV}$ infected livers.[]. Furthermore, an increase in serum CCL2 correlates with progressive liver inflammation in infected persons compared to healthy individuals [7].

Expression of CCR2 is on monocytes, T cells, DCs and macrophages. CCR2 ligands include CCL2 (also known as monocyte chemotactic protein-1, MCP-1), CCL13, CCL8 and CCL7, all of them are expressed in the hepatic environment. mRNA transcripts for CCL2 and CCR2 are significantly increased in $\mathrm{HCV}$-infected livers [ 8$]$

\section{PATIENTS AND METHODS}

\section{Study population}

This study was carried out in the Molecular Genetic Unit in Endemic Medicine \& Virological Diseases (MGEVD), Faculty of Medicine, Mansoura University, on Egyptian HCV patients and their families or close household contacts from different Egyptian populations from Dakahlia governorate between 2014 and 2015. Each family was selected on the basis of containing at least one positive $\mathrm{HCV}$ index patient, one positive HCV member and one other member who was $\mathrm{HCV}$ negative with no history of any liver complications or disorders.

A total of 75 Egyptian families were recruited in this study with a total of 291 individuals (146 infected persons with chronic hepatitis C (CHC), 145 controls (negative household contact to $\mathrm{HCV}$ : $\mathrm{HCV}$ antibody positive but HCV-RNA negative in two successive samples at least 6 months apart. The index cases in the family were selected with inclusion criteria that included HCV positive by PCR RNA for $>6$ months, adults (above 18 years) of both sexes and any stage of HCV-related liver diseases. Key exclusion criteria were as follows: patients co-infected with HIV or HBsAg, patients with anti-HCV antibodies positive and no detectable HCV-RNA in the serum.

Patients with autoimmune hepatitis, HCC and metabolic liver diseases were excluded. Healthy

control household contacts were included in this study with inclusion criteria of age $>18$ years of both sexes, first- and second-degree consanguinity to the index case living and sharing usual family activity with the index case with at least 15 years exposure to $\mathrm{HCV}$ infection and having no serological evidence of HCV, HBV or HCC and with no history of liver disease. Each participant was subjected to molecular diagnosis and HCVRNA analysis to confirm HCV infection. Written informed consent was obtained from each patient included in the study, and the study protocol conforms to the ethical guidelines of the 1975 Declaration of Helsinki as reflected in a priori approval by the institution's human research committee in Mansoura University. 


\section{Procedures}

\section{Collection of blood samples}

Three ml blood sample was withdrawn from each patient. One $\mathrm{ml}$ was delivered to $\mathrm{k}_{2}$ EDTA vacutainer tubes for DNA host analysis. The EDTA blood was then aliquoted and stored at $-50 \mathrm{C}$. The remaining blood sample was delivered into plain tubes centrifuged at $4000 \mathrm{rpm}$ for 10 min. Then the serum was separated and aliquoted into $250 \mu \mathrm{l}$ volume and stored at 50 until used.

\section{Serological markers}

Anti-HCV Abs was assessed using an ELIZA immunoassay technique (Abbott Laboratories, Abbott Park, IL, USA), quantitative PCR for positive anti $\mathrm{HCV}$ patients was done.

\section{Molecular diagnosis \\ DNA Extraction}

Genomic DNA was extracted and purified from leucocytes of peripheral blood samples using a commercial Qiagen DNA isolation kit (Qiamp® DNA Minikit, Qiagen, Germany), according to the manufacturer's instructions.

\section{Allelic discrimination with real time PCR} One Single-nucleotide polymorphisms (SNP) of the Chemokine ligand 2 (CCL2) was studied on the patients of this study the allelic genotyping of each DNA sample with real-time PCR reaction on the apparatus (model 7500; Applied Biosystems) using a ready-made fluorescein-amidite-labeled SNP primers and probes (purchased from Applied Biosystems) for one SNP of CCL2 for gene CCL2 rs13900 and TaqMan® Universal Master Mix II (2x):Supplied from (Applied Biosystems) .also DNA Template, RNase free water and optical plate (MicroAmp ${ }^{\circledR}$ Optical 96-Well Reaction Plate, Applied Biosystems), figure 1.

\section{RESULTS:}

The current study 75 Egyptian families was recruited for this study. Laboratory data are

presented in table 1. All individuals of these families (291) were genotyped for SNP CCL2 rs13900 C/T polymorphism.

Table 1 show Clinical \& laboratory Characteristics of studied groups. All samples were successfully genotyped for rs13900. Figure 2 show the distribution of the allele carriage of CCL2 rs13900 among different groups.

Table (2) and figure (2) show the distribution of allele carriage and allele frequency, Hardy Weinberg and heterozygosity of different SNPs among different groups. All groups for all SNPs are in hardy Weinberg equilibrium. $\mathrm{P}$ value is greater than 0.5 indicating that the groups of current study represent our population for genetic analysis.

The heterozygosity of all SNPs in different groups is ranged from 0.194 to 0.51 indicating that this SNPs are suitable for performing genetic analysis in the current groups.

The allele carriage of at least one copy of $\mathrm{C}$ allele of CCL2 rs13900 C/T polymorphism was significantly lower in the positive group when compared to that of negative group $(\mathrm{OR}=0.4169,95 \% \mathrm{CI} 0.1830$ to $0.9496, \mathrm{p}$ 0.0372) indicating that The $\mathrm{C}$ allele of rs $13900 \mathrm{C} / \mathrm{T}$ polymorphism is protective allele against development of chronic $\mathrm{HCV}$, Table (3).

On other hand the allele carriage of homozygotes TT of CCL2 rs13900 C/T polymorphism was significantly higher in the positive group when compared to that of negative group $(\mathrm{OR}=2.3986,95 \% \mathrm{CI}$ 1.0530 to

5.4635, $\mathrm{p}$ 0.0372) indicating that The $\mathrm{T}$ allele of rs13900 C/T polymorphism is risk 
allele for development of chronic HCV, Table (4).

\section{DISCUSSION:}

CCL2 is considered one of the member of the $\mathrm{CC}$ chemokine subfamily the responsibility for the mononuclear cells movement is due to the concentration gradient of CCL2, mainly monocytes/macrophages, to the sites of inflammation. A several cell types produce CCL2, including macrophages, lymphocytes, neutrophils, fibroblasts, keratinocytes, vascular endothelial cells and various cancer cell lines. These immune and non-immune cells produce CCL2 as a result of stimulation by a large group of mediators that include cytokines, lipopolysaccharides and growth factors referring the role of CCL2 in host defense against bacteria [8].

In HCV-infected livers, there are markedly raised in mRNA transcripts for CCL2 and CCR2. In addition, an increase in serum CCL2 associates with progressive liver inflammation in infected persons compared to healthy persons. Kupffer cells release IFN-inducible CCL2 in the early phase of infection and its secretion stimulates the first wave of infiltrating monocytes including CCR2+ plasmacytoid DCs. Also, CCR2 expressing CD8+ $\mathrm{T}$ cells are

enriched in the inflamed liver during persistent infection with HCV [9].

Genetic analysis in the current study assure the contribution of CCL2 in the Pathogenesis of $\mathrm{HCV}$. We genotype one SNP namely CCL2 rs13900 in 75 Egyptian family with at least one member affected with $\mathrm{HCV}$.

The allele frequency of the existing study are consistent with the published global frequency. In brief the allele $\mathrm{T}$ of CCL2 rs 13900 is a risk allele while the allele $\mathrm{C}$ of CCL2 rs13900 is a protective allele.
During different stages of $\mathrm{HCV}$ infection, several chemokines play sophisticated roles which may illustrate some of the conflicting findings from studies analyzing the influence of chemokine gene mutations on HCV pathogenesis.

It explain to what extent targeting chemokines therapeutically is so complex [10].

\section{REFERENCES:}

1. Koike, K., The oncogenic role of hepatitis $C$ virus, in Viruses and Human Cancer. 2014, Springer. p. 97-111.

2. El-Bendary, M., et al., Epidemiological aspects of intrafamilial spread of $\mathrm{HCV}$ infection in Egyptian population: a pilot study. Open Journal of Gastroenterology, 2014. 4(05): p. 228.

3. Viola, A. and A.D. Luster, Chemokines and their receptors: drug targets in immunity and inflammation. Annu. Rev. Pharmacol. Toxicol., 2008. 48: p. 171-197.

4. Zlotnik, A. and O. Yoshie, Chemokines: a new classification system and their role in immunity. Immunity, 2000. 12(2): p. 121-127.

5. Kehrl, J.H., Chemoattractant receptor signaling and the control of lymphocyte migration. Immunologic research, 2006. 34(3): p. 211-227.

6. Mellado, M., et al., Chemokine signaling and functional responses: the role of receptor dimerization and TK pathway activation. Annual review of immunology, 2001. 19(1): p. 397-421.

7. Eberlein, J., et al., Comprehensive assessment of chemokine expression profiles by flow cytometry. The Journal of clinical investigation, 2010. 120(3): p. 907.

8. Fahey, S., E. Dempsey, and A. Long, The role of chemokines in acute and chronic hepatitis $C$ infection. Cellular and Molecular Immunology, 2014. 11(1): p. 25-40. 
9. Heydtmann, M. and D.H. Adams, Chemokines in the immunopathogenesis of hepatitis C infection. Hepatology, 2009. 49(2): p. 676-688.
10. Boisvert, J., et al., Liver-infiltrating lymphocytes in end-stage hepatitis $C$ virus: subsets, activation status, and chemokine receptor phenotypes. Journal of hepatology, 2003. 38(1): p. 67-75.
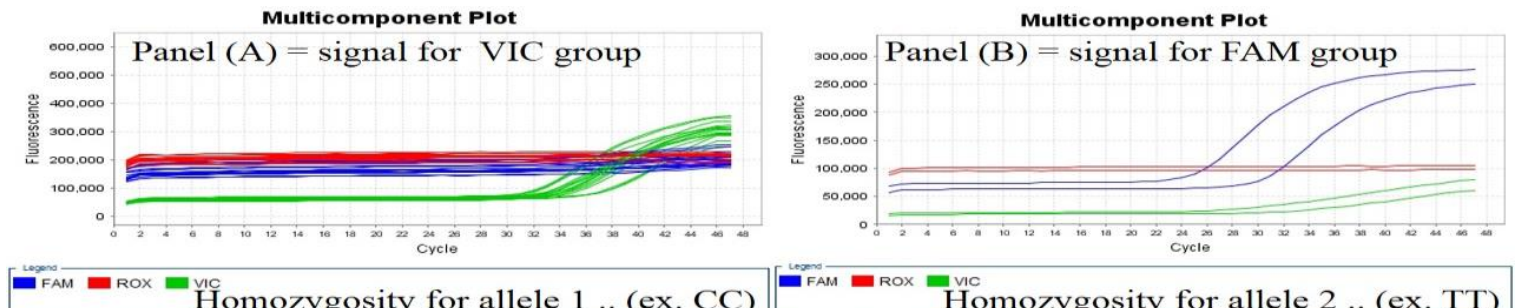

Homozygosity for allele 2 ... (ex. TT)

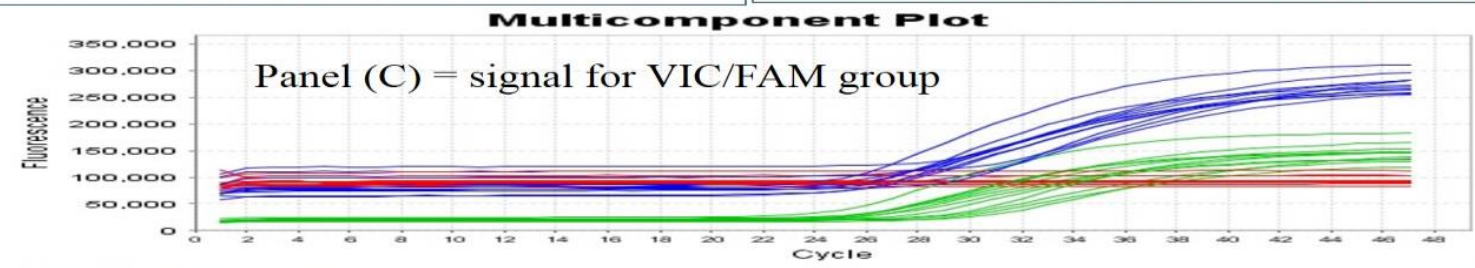

Heterozygosity for both alleles .. (ex. CT)

Figure 1: Real Time PCR charts for different genotypes of (rs 13900 C/T) SNP of CCL2 gene

Life Technologies follows the pattern of assigning FAM ${ }^{\mathrm{TM}}$ dye as the reporter for the target assay and assigning VIC ${ }^{\circledR}$ dye as the reporter for the normalizer assay. (Applied Biosystems provides common endogenous control assays for human, mouse, and rat that are available primer-limited and labeled with the VIC® dye, which is recommended for multiplexing with FAM $^{\mathrm{TM}}$ dye). The allele specific probes were labeled with a fluorescent dye (VIC and FAM) and used in the allele typing of each DNA sample with real-time PCR reaction on the apparatus (model 7500; Applied Biosystems) using a readymade fluorescein-amidite-labeled SNP primers and probes.

Heterozygosity: having two different alleles of the same gene.

Homozygosity: having two identical alleles of the same gene. 


\section{Distribution of the allele carriage of CCL2 rs13900 among different groups}

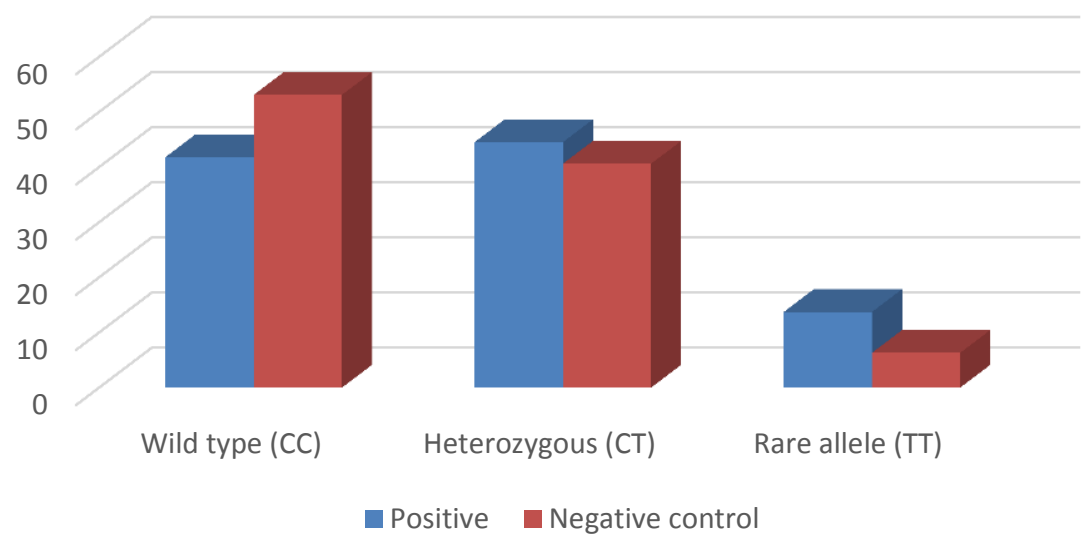

Figure 2: Distribution of the allele carriage of CCL2 rs13900 among different groups

Table 1: Clinical \& laboratory Characteristics of studied groups

\begin{tabular}{|c|c|c|}
\hline & Positive (146) & Negative (145) \\
\hline Sex: M /F & $84 / 62$ & $62 / 83$ \\
\hline & Mean $( \pm \mathrm{SD})$ & Mean $( \pm$ SD) \\
\hline Age & $39.5(15.97)$ & $33.2(23.9)$ \\
\hline S. Bilirubin (mg/dl) & $1.4(0.68)$ & $0.8(0.2)$ \\
\hline S. Albumin (gm/dl) & $3.8(0.6)$ & $4.6(0.5)$ \\
\hline SGOT (IU/L) & $45(11)$ & $19.0(6)$ \\
\hline SGPT & $52(11)$ & $20.0(8)$ \\
\hline ALP U/L & 77.8 (15.9) & $48.0(18.0)$ \\
\hline AFP $n g / m L$ & $25(16.8)$ & $2.5(2.5)$ \\
\hline ANA ( U) & $0.59(0.20)$ & $0.6(0.25)$ \\
\hline S. Creatinine & $0.8(0.35)$ & $0.8(0.39)$ \\
\hline
\end{tabular}

S. : Serum, S. Bilirubin (N: <1.1 mg/dl); S. Albumin (N 3.9-5.1 gm/dl); SGOT (aspartate aminotransferase) (N< $40 \mathrm{IU} / \mathrm{L}) ; \quad(\mathrm{SGPT})$ alanine aminotransferase $(\mathrm{N}<45 \mathrm{IU} / \mathrm{L}) ; \quad$ ALP $=$ Alkaline phosphatase $(\mathrm{N}: 37-116 \mathrm{U} / \mathrm{L})$; Alpha Fetoprotein (AFP) $(\mathrm{N}<10 \mathrm{ng} / \mathrm{ml})$ : Antinuclear Antibodies (ANA) $(\mathrm{N}<1.1 \mathrm{U})$; S. creatinine $(\mathrm{N}<1.4 \mathrm{mg} / \mathrm{dl})$;

S. Cholesterol $(\mathrm{N}<200 \mathrm{mg} / \mathrm{dl})$ 
Table 2: Allele carriage and allele frequency of CCL2 rs13900 among different groups

\begin{tabular}{|l|c|c||}
\hline & Negative control (145) & Positive (146) \\
\hline \hline Wild type (CC) & $77(53.1 \%)$ & $61(41.8 \%)$ \\
\hline Heterozygous (CT) & $59(40.6 \%)$ & $65(44.5 \%)$ \\
\hline \hline Rare allele (TT) & $9(6.2 \%)$ & $20(13.7 \%)$ \\
\hline \hline Allele frequencies & 0.734 & 0.640 \\
C & 0.266 & 0.360 \\
T & 0.334 & 0.349 \\
\hline \hline PIC & 0.407 & 0.442 \\
\hline \hline Heterozygosity & 290 & 292 \\
\hline No of alleles & 3.66981607 & 2.672123425 \\
\hline \hline Hardy-Weinberg & 0.055406 & 0.102120 \\
x2 & & \\
P & & \\
\hline
\end{tabular}

PIC: Polymorphism information content.

Table 3: The risk of allele C of CCL2 rs13900 carriage

\begin{tabular}{|l|c|}
\hline & Positive vs. negative \\
\hline Odds ratio (OR) & 0.4169 \\
\hline 95\% CI & 0.1830 to 0.9496 \\
\hline z statistic & 2.083 \\
\hline P & 0.0372 \\
\hline
\end{tabular}

95\% CI: Confidence intervals

Table 4: The risk of allele TT of CCL2 rs13900 carriage

\begin{tabular}{|l|c|}
\hline & Positive vs. negative \\
\hline Odds ratio (OR) & 2.3986 \\
\hline 95\% CI & 1.0530 to 5.4635 \\
\hline $\mathbf{z}$ statistic & 2.083 \\
\hline $\mathbf{P}$ & 0.0372 \\
\hline
\end{tabular}

